\title{
4 Die jüdische Sportbewegung im Wien der Zwischenkriegszeit
}

Betrachtet man die jüdischen Sportvereine Wiens der Zwischenkriegszeit, dann bilden sich in ihnen alle Facetten der jüdischen Gemeinschaft der Donaumetropole ab. Eine genuin jüdische Sportbewegung hat ihre Wurzeln im ausgehenden 19. Jahrhundert und war Reaktion auf eine immer massiver werdende Ausgrenzung von jüdischen Aktiven und Funktionären in den bestehenden Vereinen. Als 1887 der Erste Wiener Turnverein einen Arierparagrafen in seine Vereinsstatuten aufnahm und damit etwa 50 Prozent der Mitglieder den Verein verlassen mussten $^{1}$, gründeten ausgeschlossene wie mit der Entwicklung unzufriedene Mitglieder den Deutsch-österreichischen Turnverein Wien. Zeitgleich begann 1899 mit der Gründung des Turnvereins jüdischer Hochschüler - ab 1900 nannte er sich Erster Wiener Jüdischer Turnverein - eine Öffnung über den akademischen Bereich hinaus. Sie stand am Anfang einer Entwicklung, die ihre Fortsetzung in weiteren jüdischen Makkabi-Turnvereinen und seinen Höhepunkt in der Geschichte des Sportclub Hakoah finden sollte.

\section{Die Anfänge jüdischer Partizipation im Wiener Sport}

Ebenfalls zu Ende des 19. Jahrhunderts begannen jüdische Aktive eine wichtige Rolle bei den ersten Allroundsport-Vereinsgründungen zu spielen, etwa beim Wiener Athletiksport Club (WAC) oder beim First Vienna Football Club 1894. ${ }^{2}$ Beispielhaft dafür ist der Schwimmer Otto Herschmann zu erwähnen, der an den Olympischen Spielen 1896 in Athen teilnahm, wo er über 100 Meter Freistil den zweiten Platz erreichte. Beeindruckt von den Leistungen der Sportler in Athen, war er gemeinsam mit dem jüdischen Industriellen August Wärndorfer

1 Matthias Marschik, Von jüdischen Vereinen und „Judenclubs“. Organisiertes Sportleben um die Jahrhundertwende, In: Adunka, Lamprecht, Traska (Hg.), Jüdisches Vereinswesen, 225-244, hier 228.

2 Zum WAC siehe Alexander Juraske, Der Wiener Athletiksport-Club und seine jüdischen Mitglieder, in: David, Jüdische Kulturzeitschrift, 111 (Dezember 2016) $42 \mathrm{f}$. Zur Vienna siehe Alexander Juraske, Blau-Gelb ist mein Herz. Die Geschichte des First Vienna Football Club 1894 (Wien 2017) 73-76.

Ә Open Access. (c) 2019 Alexander Juraske, publiziert von De Gruyter. (cc) BY Dieses Werk ist lizenziert unter der Creative Commons Attribution 4.0 International Lizenz (CC BY 4.0). 
führend an der Gründung des WAC beteiligt, bei dem er in der Folge in unterschiedlichen Positionen tätig war. Über seine Vereinszugehörigkeit hinaus engagierte sich der spätere promovierte Jurist in der Institutionalisierung des österreichischen Sportlebens. Zusammen mit Wärndorfer saß Herschmann im Exekutivkomitee des Wiener Comité zur Beschickung der Pariser Olympischen Spiele im Jahre 1900. ${ }^{3}$ Als Vertreter des WAC war er an der Gründung des Österreichischen Sportausschusses für leichte Athletik, einem Vorgänger des Leichtathletikverbandes, beteiligt. ${ }^{4}$ Ferner wurde er Präsident des Österreichischen Zentralverbands für gemeinsame Sportinteressen, dem Vorläufer des Österreichischen Olympischen Comités (ÖOC). ${ }^{5}$ Auch als ÖOC-Präsident war der beruflich als Hof- und Gerichtsadvokat tätige Herschmann als Fechter, Ringer, Schwimmer und Wasserballer aktiv. 1912 errang er mit der Säbelmannschaft bei den Olympischen Spielen in Stockholm eine Silbermedaille und ist damit der einzige Präsident eines nationalen Olympischen Komitees, der während seiner Amtszeit eine Medaille gewinnen konnte. 1914 trat er von der Spitze des ÖOC ab und wurde wenig später Vorsitzender des Verbandes Österreichischer Schwimmvereine. ${ }^{6}$ Ferner war er auch im Fechtverband aktiv.

Darüber hinaus setzte sich Herschmann auch theoretisch mit der jüdischen Partizipation im österreichischen Sport auseinander. Dieser Thematik widmete er in seinem Buch „Wiener Sport“ ein eigenes Kapitel. Die Stellung von Juden im österreichischen Sportbetrieb seiner Zeit skizziert er so, „daß sie in den älteren Sportzweigen, deren Bestand auf längere Frist als zehn Jahre zurückreicht, fast gar keine oder keine besondere, in den völlig modernen Sportzweigen hingegen eine bedeutende, vielfach sogar eine tonangebende Rolle spielen. “7 Er fasst zusammen, „daß die Juden im Rudern, in der schweren Athletik, im Eislaufen und im Radfahren teilweise überhaupt nicht $\mathrm{zu}$ finden waren, teilweise verhältnismäßig höchst minderwertige Leistungen zeigten, daß sie hingegen im Fußball, im italienischen Fechten, im Tennis, in der leichten Athletik und zumal im Schwimmen eine ausgezeichnete Position innehaben und, ja, daß ihnen in einigen dieser Sporte die Führung rückhaltlos zuerkannt werden muß."8

Das jüdische Engagement in bestimmten Sportarten sah Herschmann nicht allein darin begründet, dass ihnen ,in verschiedenen Sportzweigen, welche in

3 Allgemeine Sport-Zeitung (6.1.1900) 16.

4 Allgemeine Sport-Zeitung (15. 9. 1900) 1032.

5 Allgemeine Sport-Zeitung (17. 12. 1911) 1713.

6 Allgemeine Sport-Zeitung (7. 3 1915) 135.

7 Otto Herschmann, Wiener Sport (Großstadt Dokumente) (Berlin/Leipzig 1904) 22.

8 Herschmann, Sport, $22 \mathrm{f}$. 
einer bestimmten eng umgrenzten Gesellschaft betrieben wurden, einfach den [sic] Zutritt versperrt“ war, vielmehr „suchten sie selbst in ihrem wohl begreiflichen Betreiben, ihre Kraft neuen Unternehmungen zuzuwenden“, aktiv sportliche Betätigungsfelder. ${ }^{9}$ Als Verfechter einer jüdischen „Assimilation“ schrieb er, „daß das Aufgehen des Judentums in den Volkskörpern, unter welchen es lebt, die einzige richtige Lösung der Judenfrage ist [...] wo all jene Wege und Mittel bedacht werden müssen, welche diese Lösung beschleunigten und erleichtern, wird man auch des Sportes als Mittel zu diesem Zwecke nicht entraten dürfen.“ So ließe sich der Sport ,als kosmopolitisches Einigungsmittel gebrauchen und als solches auch im Dienste der Assimilation der Juden verwenden." 10

Parallel zur Mitwirkung von Juden bei den Gründungen der ersten Allroundsportvereine waren jüdische Aktive bei der Etablierung des jungen Fußballsports in Wien zunächst an der Gründung des - allerdings kurzlebigen Vereins Olympia beteiligt, ehe sich 1897 - unter großer Beteiligung seiner jüdischen Mitglieder - innerhalb des Deutsch-österreichischen Turnvereins Wien eine Fußballsektion formierte, die sich unmittelbar nach dem Kaiser-Franz-Joseph-Jubiläums-Turnier als Wiener Fußballclub 1898 selbstständig machte. ${ }^{11}$

1899 startete die genuin jüdische Sportbewegung, als mit dem Turnverein jüdischer Hochschüler, als Reaktion auf Ausgrenzungsmechanismus und als Resultat eines gesteigerten jüdischen Selbstwertgefühls, der erste jüdische Sportverein in Wien gegründet wurde. Ein Jahr später benannte sich der Verein um in Erster Wiener Jüdischer Turnverein (ab 1914 mit Namenszusatz Makkabi IX) und öffnete sich über den akademischen Bereich für Nicht-Hochschüler und Frauen. ${ }^{12}$ Mit dem Turnverein Zion - dem späteren Jüdischen Turnverein Makkabi XV - bildete sich 1900 ein weiterer jüdischer Turnverein in Wien. Der Entwicklung in Wien folgend, entstand 1905 in der steirischen Landeshauptstadt der Jüdische Turnverein zu Graz, der spätere Jüdische Turnverein Makkabi Graz. ${ }^{13}$ Nachdem dessen Vereinsaktivitäten im Ersten Weltkrieg zum Erliegen kamen, fanden viele jüdische Sportler und Sportlerinnen im 1919 gegründeten Sportklub Hakoah Graz eine neue sportliche Wirkungsstätte.

9 Herschmann, Sport, 23.

10 Herschmann, Sport, 26.

11 Allgemeine Sport-Zeitung (24. 9. 1898) 1157.

12 Marschik, Vereine, 229.

13 Heimo Halbrainer, „Keine ausschließliche Turn- und Sportbewegung“. Jüdischer Sport in der Steiermark am Beispiel des Jüdischen Turnvereins „Makkabi“ und der Hakoah. In: Gerald Lamprecht (Hg.), Jüdisches Leben in der Steiermark. Marginalisierung - Auslöschung - Annäherung (Schriften des Centrums für Jüdische Studien 5, Innsbruck/Wien/Bozen 2004) 171-189, hier 176. 
Schnell wurde der Allroundsportverein zum Sammelbecken jüdische Sportler und Sportlerinnen in der steirischen Landeshauptstadt ${ }^{14}$ und zum größten jüdischen Verein außerhalb Wiens.

Für die jüdische Sportbewegung in Österreich, wie in vielen anderen europäischen Ländern auch, lieferte der Zionist Max Nordau mit seinem Konzept vom „Muskeljudentum“, das er zum ersten Mal in einer Rede auf dem Zweiten Zionistenkongress 1898 in Basel entwarf, ein theoretisches Fundament, das konträr zu den assimilatorischen Vorstellungen von Otto Herschmann stand. ${ }^{15}$ Dem verbreiteten antisemitischen Vorurteil des „körperlich schwachen Juden“ begegnete der zionistische Vordenker mit dem Archetypus des „neuen starken jüdischen Menschen“: „Wir haben von Natur die unerläßlichen geistigen Vorbedingungen außergewöhnlicher athletischer, turnerischer Leistungen. Die körperlichen Voraussetzungen, ein gewisses Maß an Muskelstärke, sind durch Übung zu erlangen. Wir haben also alles, was nötig ist, um uns als Turner ebenso glänzend zu bewähren wie als Pfleger aller Geistesdisziplinen.“16

Den modernen Sport lehnte Nordau hingegen, aufgrund seines Strebens nach Preisen und Rekorden, als egoistisch und materialistisch ab. Nur dem Turnen sprach er die Qualität zu, eine „harmonische Ausbildung“ des Körpers sicherzustellen, die sowohl dem Einzelnen wie auch dem Kollektiv zugute kommen sollte. ${ }^{17}$ Neben der Arbeit am Körper selbst forderte er auch eine Sichtbarmachung der jüdischen Sportaktivitäten. Mit dem Davidstern auf dem Trikot oder den Initialen ihrer jüdischen Vereine sollten jüdische Sportler und Sportlerinnen selbstbewusst in der Öffentlichkeit auftreten. ${ }^{18}$ Indem Nordau seine Ausführungen auf dem Fünften Zionistenkongress in Wien im Jahr 1901 wiederholte, beeinflusste er auch die Entwicklung in Wien nachhaltig. In der Folge gründeten sich weitere jüdische Turnvereine in Wien, sodass 1913 schon sechs jüdische Turnvereine bestanden. ${ }^{19} 1910$ entstand der Sportklub jüdischer Studenten. ${ }^{20}$

Der Verein, der die Ideen Nordaus in Wien auf den allgemeinen Sportbereich ausweitete, war der 1909 gegründete Sportclub Hakoah. Selbstbewusst

14 Halbrainer, Sportbewegung, 178.

15 Daniel Wildmann, Muskeljuden, turnende Juden und moralische Juden. In: Dachs (Hg.), Jüdischer Almanach, 103-122, hier 103.

16 Zionistisches Aktionskomitee (Hg.), Max Nordau's zionistische Schriften (Köln/Leipzig 1909) 387.

17 Wildmann, Muskeljuden, 111.

18 Wildmann, Muskeljuden, 108.

19 John Bunzl (Hg.), Hoppauf Hakoah. Jüdischer Sport in Österreich. Von den Anfängen bis in die Gegenwart (Wien 1987) 16.

20 Allgemeine Sport-Zeitung (1. 1. 1910) 20. 


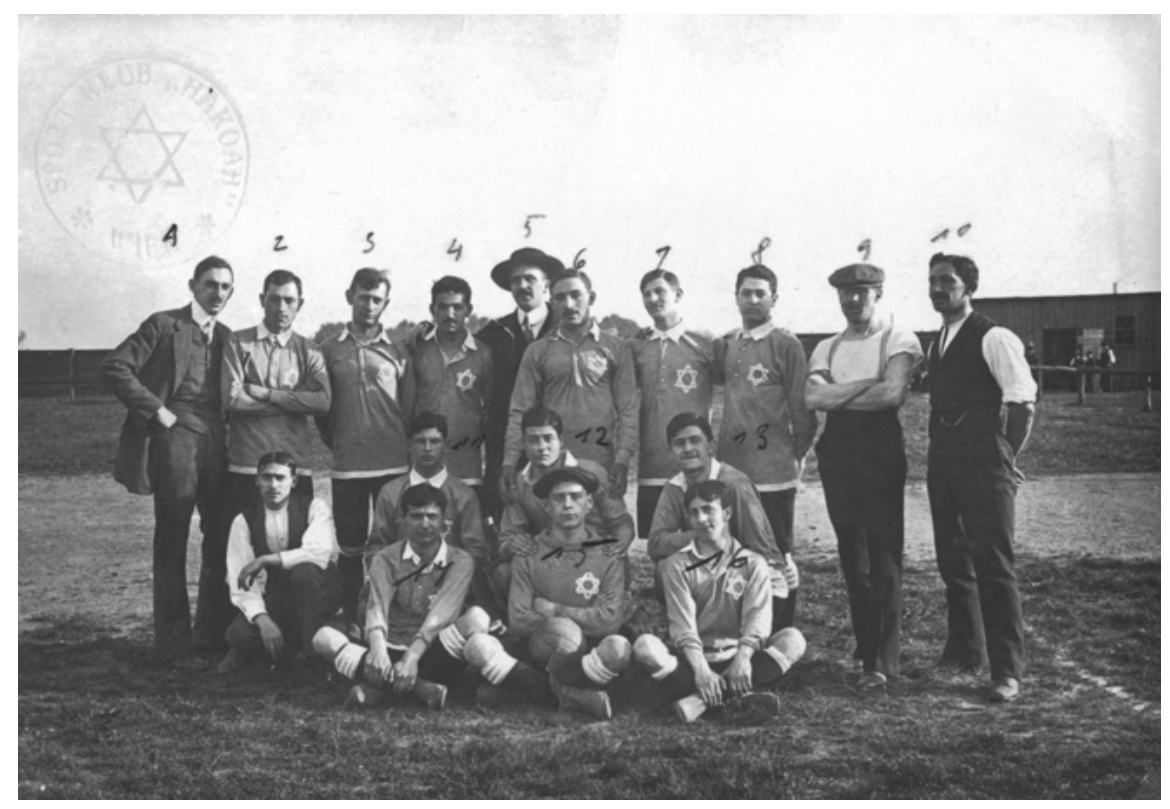

Abb. 5: Fußballteam der Hakoah, ca. 1922. Links außen: Sektionsleiter Arthur Baar, Mitte mit Hut: Fritz Beda-Löhner (Pierre Gildesgame Maccabi Sports Museum).

traten die Aktiven der zionistischen Hakoah mit dem Davidstern auf der Brust in der Öffentlichkeit auf und feierten gerade in den publikumswirksamen Sportarten - wie etwa im Fußball, aber auch im Schwimmsport oder der Leichtathletik - große Erfolge.

Der SC Hakoah sah sich als Sammelbecken für alle jüdischen Sportler und Sportlerinnen, die Ausgrenzung und Antisemitismus in den nichtkonfessionellen Vereinen erfahren hatten, und förderte darüber hinaus durch Vorträge und weitere Veranstaltungen das jüdische Selbstbewusstsein. ${ }^{21}$ Wer Mitglied werden wollte, musste Jude bzw. Jüdin sein. Nichtjuden waren nur als Trainer bzw. Trainerin erlaubt. ${ }^{22}$ Zwar war die Hakoah aus dem eigenen Selbstverständnis heraus ein zionistischer Verein. Es wäre aber zu kurz gegriffen, alle seine Mitglieder als Zionisten oder Zionistinnen zu bezeichnen. Einerseits stand die sportliche Betätigung, die oft mit der politischen Einstellung konform ging, im Vordergrund der Vereinsaktivitäten. Gleichzeitig war der Verein selbst aber mehr als nur ein Sportverein. Gerade Juden und Jüdinnen, die den politischen

21 Bunzl, Hoppauf, 24.

22 Bunzl, Hoppauf, 25. 
Zielen des Zionismus skeptisch oder ablehnend gegenüberstanden, konnten partizipieren bzw. sich als Anhänger bzw. Anhängerinnen der Hakoah fühlen, weil der Verein allein schon durch seine weitreichende Tätigkeit das jüdische Selbstwertgefühl im besonderen Maße hob.

Im Gegensatz zu den jüdischen Turnvereinen positionierte sich die Hakoah schon früh als Allroundsportverein und hatte regen Zulauf. Schon zehn Jahre nach der Gründung betrieben über 1.000 Mitglieder Sport in den Sektionen Fußball, Schwimmen, Leichtathletik, Tennis, Ski und Touristik und Schwerathletik. ${ }^{23}$ Neben den Sportaktivitäten entwickelte sich ein breitgefächertes Kulturleben mit Vorträgen, Festveranstaltungen und Kabarettabenden - etwa die Hakoah-Redoute sowie die „Beda“-Abende mit dem Librettisten und HakoahPräsidenten Fritz Löhner-Beda. Diese kulturellen Aktivitäten mündeten auch in die Gründung eines eigenen Vereinsorchesters sowie einer Tanzsektion. ${ }^{24}$ Selbst der Ausbruch des Ersten Weltkrieges konnte das Vereinsleben nur kurzfristig beeinträchtigen.

\section{Die Blütezeit jüdischer Vereine in Wien}

Ab Herbst 1918 nahm allgemein die Sportbegeisterung in Wien zu, was sich in einem Vereinsgründungsboom, besonders im Fußballsport, niederschlug. ${ }^{25}$ Den ehemals auf bestimmte Schichten begrenzten Fußball hatten alle männlichen Bevölkerungsschichten im Ersten Weltkrieg an der Front oder in der Etappe kennen- und schätzen gelernt. Zurück in der Heimat nahm der Fußball gerade unter den Arbeitern einen enormen Aufschwung und institutionalisierte sich schnell im Arbeiterfußball. Allgemein stiegen sowohl die Zahl der Aktiven als auch der Zuschauer rapide an. ${ }^{26}$

Dieser allgemeine Aufschwung erfasste auch die jüdische Sportbewegung und neben der arrivierten Hakoah und den jüdischen Turnvereinen kam es zu einer Fülle an weiteren jüdischen Vereinsgründungen. Dabei war die soziopolitische Bandbreite unter den neuen jüdischen Vereinen sehr hoch. Allroundsportvereine wie Kadimah, Hechawer und Hasmonea waren stark zionistisch geprägt, standen der Hakoah nahe und kooperierten mit dem jüdischen Aushängeschild. Kleinere Vereine wie Achduth, Achim, Ari und Hagibor versuch-

23 Wiener Morgenzeitung (1. 8. 1919) 6.

24 Zur Tanzsektion siehe Jüdische Zeitung (15. 12. 1911) 6.

25 Matthias Marschik, Vom Herrenspiel zum Männersport: Modernismus - Meisterschaft Massenspektakel. Die ersten Jahre Fußball in Wien (Wien 1997) 142.

26 Marschik, Herrenspiel, 142. 
ten sich stark auf den Fußballsport zu konzentrierten. ${ }^{27}$ Andere Vereine spezialisierten sich auf andere Sportarten. So bildeten ehemalige Aktive der Hakoah den Hockeyklub „Blau-Weiß“. Borochow, benannt nach einem zionistischen Vordenker, legte seinen Fokus auf den Schachsport und (Makkabi) Hazair konzentrierte sich auf die Leichtathletik. ${ }^{28}$ Neben den zionistischen Vereinen gründeten sich auch andere jüdische Klubs. Mit der kurzlebigen Hanizachon formierte sich ein orthodoxer Sportverein. ${ }^{29}$ Der Jüdische Athletik Club (J.A.C.) und der Young Jewish Sporting Club standen wiederum der Wiener Sozialdemokratie nahe. ${ }^{30}$

Anfang der 1920er-Jahre hatte also der Sportboom, besonders im Fußballsport, eine jüdische Vereinsgründungswelle ausgelöst. Diese Tatsache war dem verstärkten Zuzug osteuropäischer Juden in die Reichshauptstadt im Ersten Weltkrieg geschuldet, die aus den ehemaligen k. u. k. Regionen sowie aus dem Gebiet Russlands, vor unsicheren Lebensbedingungen und Verfolgung geflohen waren.

Viele der Vereine, die zionistisch geprägt waren, nahmen bei ihrer Namensfindung Bezug auf die Helden der jüdischen Geschichte: mit Makkabi auf den Makkabäeraufstand im zweiten Jahrhundert vor unserer Zeitrechnung, mit Bar Kochba auf den Anführer des Aufstandes gegen die Römer oder mit „Hasmonea“ nach der Hasmonäer-Dynastie, aus der die Makkabäer stammten. ${ }^{31}$ Auch „Massada“ (JTV Massada, Makkabi XVII) gehört dazu, benannt nach der Bergfestung, in der die jüdischen Aufständischen um Eleasar ben Ja'ir 73 nach unserer Zeitrechnung den Römern zu trotzen versuchten. ${ }^{32}$ Die Zionisten suchten so an das in ihren Augen ,authentische“ prä-diasporische Judentum der Antike vor der Zerstörung Judäas durch die Römer anzuknüpfen. ${ }^{33}$ Neben der historischen Bezugnahme wählten Vereine aber auch Namen wie Hagibor (Der Held) oder - bei linken Vereinen - Hapoel (Der Arbeiter). ${ }^{34}$

Als Dachorganisation, die die Interessen der jüdischen Vereine wahrnehmen sollte, konstituierte sich innerhalb des 1903 gegründeten Makkabi-Welt-

27 Marschik, Von jüdischen Vereinen, 234.

28 Marschik, Von jüdischen Vereinen, 234.

29 Wiener Morgenzeitung (25. 2. 1922) 9.

30 Marschik, Von jüdischen Vereinen, 234.

31 Michael Brenner, Keine jüdische Geschichte des zwanzigsten Jahrhunderts ohne Sport. In: Dachs (Hg.), Jüdischer Almanach, 12-22, hier 13.

321912 gründete sich der Jüdische Turnverein Massada - später Jüdischer Turnverein Makkabi 17, siehe Jüdische Monatshefte für Turnen und Sport, 1913, H. 7, $224 \mathrm{f}$.

33 Moshe Zimmermann, Der Sport unterwegs nach Palästina - jüdisch, zionistisch, deutsch? In: Jutta Fleckenstein, Lisa Maria Tillian-Fink (Hg.), Never Walk Alone. Jüdische Identitäten im Sport (Berlin 2017) 175-181, hier 177.

34 Brenner, Sport, 13. 
verbands der Kreis Deutschösterreich der jüdischen Turn- und Sportvereine unter ihrem Vorsitzenden Dr. Heinrich Schiffmann und seinem Stellvertreter Adolf Taglicht. ${ }^{35}$ Aufgrund der unterschiedlichen Interessen von Turn- und Sportvereinen bildeten sich rasch eigene Unterausschüsse für „Turnen“ und „Sport“. In Letzterem waren 1922 schon 14, zumeist sehr junge, Vereine zusammengefasst. ${ }^{36}$ Doch der Interessensgemeinschaft, in der zum Großteil zionistische Vereine vertreten waren, blieben die dem Arbeitersport nahestehenden Klubs fern, was indirekt zu einer Schwächung der Dachorganisation beitrug.

Die Hakoah versuchte die kleineren jüdischen Vereine infrastrukturell zu unterstützen. So trugen die Fußballabteilungen von Kadimah und Hechawer ihre Heimspiele auf dem von der Hakoah gemieteten „Birner“-Platz in Floridsdorf aus. ${ }^{37}$ Während die kleineren jüdischen Vereine im Aufbau begriffen waren, feierte die Fußballabteilung der Hakoah mit der Vizemeisterschaft in der Saison 1921/22 ihren ersten großen Erfolg. In der gleichen Spielzeit war ihre Fußballsektion an Mitgliedern schon so überlaufen, dass keine neuen Aktiven aufgenommen werden konnten. So fand der damals 13-jährige Friedrich Ephraim Kantor-Berg, der spätere Schriftsteller Friedrich Torberg, nur Aufnahme in der Schwimmsektion und reüssierte später erfolgreich als Wasserballer. ${ }^{38}$ Wer Anfang der 1920er-Jahre einer jüdischen Fußballmannschaft beitreten wollte, musste mit einem der unterklassigen Vereine vorliebnehmen. ${ }^{39}$

Die Vielzahl der neuen jüdischen Vereine wurde im Fußballsport in die unteren Spielklassen eingereiht. Dort wurden ihre Aktiven und Funktionäre, nicht anders als bei der Hakoah, immer wieder Ziel von antisemitisch motivierten Schmähungen, die nicht selten zu tätlichen Angriffen und in der Folge zu Spielabbrüchen führten, wie bei den Begegnungen J.A.C. gegen SC Orkan ${ }^{40}$ oder Kadimah versus SC Matzleinsdorf ${ }^{41}$.

\section{Stagnation}

1920 gründete sich der Sportklub Hasmonea, der umgehend in den Fußballverband aufgenommen wurde. ${ }^{42}$ Hinter der Hakoah entwickelte sich der Klub zum

35 Jüdische Zeitung (27. 6. 1919) 6.

36 Wiener Morgenzeitung (15. 3. 1922) 7.

37 Wiener Morgenzeitung (29. 1. 1919) 7.

38 Michael Brenner, Kleine jüdische Geschichte (München 2012) 278.

39 Friedrich Torberg, Warum ich stolz bin. In: Dachs (Hg.), Jüdischer Almanach, 59-67, hier $59 \mathrm{f}$.

40 Wiener Morgenzeitung (24. 6. 1927) 7.

41 Wiener Morgenzeitung (5.10.1926) 8.

42 Sport und Spiele (4. 12. 1920) 313. 
wichtigsten jüdischen Allroundverein in Wien. Neben dem Fußballsport wurden Sektionen für Handball, Leichtathletik, Tischtennis und eine Kultur- und Geselligkeitsabteilung unterhalten. Auf ihre Initiative wurde mit dem „Jüdischen Cup“ auch ein eigener Wettbewerb für jüdische Vereine eingerichtet. ${ }^{43}$

Die sich schon früh anbahnenden Auffassungsunterschiede zwischen jüdischen Turnvereinen und den anderen Sportvereinen, die zumeist im Fußballbereich tätig waren, führten zur Gründung des Jüdischen Sportverbandes durch 17 Vereine unter Schriftführer Emanuel Fiscus vom SK Hasmonea und Kassier Adolf Wirtschafter von Hechawer. ${ }^{44}$ Diese neue Interessensgemeinschaft sollte allen jüdischen Vereinen bundesweit offenstehen. Sie versuchte die jüdische Sportbewegung zu stärken, indem sie ideologische und politische Differenzen überbrücken wollte. Neben den zionistischen Wiener Vereinen finden sich unter den Gründungsmitgliedern auch der Jüdische Arbeitersportklub Borochow, die Grazer Hakoah oder der Jüdische Turn- und Sportverein Linz. ${ }^{45}$ Der Versuch einer stärkeren Zusammenarbeit war auch notwendig geworden, drohten doch die jüdischen Vereine in der Auseinandersetzung zwischen dem sozialdemokratischen Verband der Arbeiter- und Soldatensportvereinigungen (VAS), ab 1924 Arbeiterbund für Sport und Körperkultur in Österreich (ASKÖ), und den bürgerlichen Vereinen aufgerieben $\mathrm{zu}$ werden. In dieser Auseinandersetzung suchte der Jüdische Sportverband letztlich eine neutrale Position einzunehmen, auch wenn es aufgrund von Naheverhältnissen mancher Mitgliedsvereine zur einen oder anderen Seite immer wieder zu strategischen Bündnissen in Einzelfragen kam.

Anfang der 1920er-Jahre existierten 24 jüdische Vereine, vornehmlich Fußballklubs in der Leopoldstadt und der Brigittenau, den beiden Wiener Bezirken mit dem höchsten jüdischen Bevölkerungsanteil. Diese Vereinsdichte führte dazu, dass die Wechselmodalitäten geklärt werden mussten, griffen doch alle Vereine in ihrer Beschränkung auf jüdische Aktive auf das gleiche Spielerreservoir zurück. So erließ der Jüdische Verband zunächst ein Wechselverbot zwischen den einzelnen jüdischen Vereinen. ${ }^{46}$ Man sah sich zu diesem Schritt veranlasst, weil man fürchtete, die Hakoah könne die besten Spieler der kleineren Vereine abwerben und so deren Entwicklung nachhaltig bedrohen. Doch wegen des eklatanten Leistungsunterschiedes zeigte sich sehr schnell, dass die Hakoah im beginnenden Professionalismus ihren Bedarf an Verstärkungen pri-

43 Wiener Morgenzeitung (18. 6. 1921) 6.

44 Wiener Morgenzeitung (22. 12. 1921) 7.

45 Zur Situation in Linz siehe Michael John, Jüdische Vereine in Linz. In: Adunka, Lamprecht, Traska (Hg.), Jüdisches Vereinswesen, 157-182.

46 Wiener Morgenzeitung (2. 2. 1923) 9. 
mär im benachbarten Ausland stillte. Bald erwies sich die Wechselbeschränkung als überaus nachteilig, wurde dadurch doch Nachwuchsspielern der Hakoah, denen der Sprung in die erste Mannschaft verwehrt blieb, ein Wechsel $\mathrm{zu}$ den kleineren jüdischen Vereinen unmöglich gemacht. So ging man vom Transferverbot ab. Trotzdem diktierte der Jüdische Sportverband harte Bedingungen für etwaige Spielerübertritte. In der Folge sollte sich zeigen, dass eher Spieler, die für die erste Mannschaft nicht infrage kamen, von der Hakoah zu den kleineren Vereinen wechselten als umgekehrt. ${ }^{47}$

Außerdem standen die kleineren jüdischen Vereine, wie viele andere Vereine auch, unter erheblichem ökonomischen Druck. Ihre Funktionäre und Aktiven kamen aus den unteren Schichten der Bevölkerung und den Vereinen fehlten die entsprechenden Gönner oder Sponsoren. Auch verfügten sie nicht über ausreichende Einnahmen durch Kartenverkäufe, da sich das jüdische Publikumsinteresse auf die Spiele der erstklassigen Hakoah konzentrierte und wenig Raum für die Kleineren ließ. ${ }^{48}$ Ferner verfügten die kleineren Vereine über keine eigenen Spielstätten. Die notwendige Anmietung fremder Plätze führte zu finanziellen Belastungen, die mitunter durch Mitgliedsbeiträge allein nicht gedeckt werden konnten. Gleichzeitig sahen sich die Aktiven und Sympathisanten der kleineren Vereine gerade auf fremden Plätzen immer wieder antisemitischen Verunglimpfungen ausgesetzt. ${ }^{49}$ Womit die Hakoah bei den Spielen auf höchster Ebene konfrontiert war, erlebten auch die unterklassigen Vereine und in den niederen Klassen waren Schutzmöglichkeiten nicht existent bzw. konnten wenige Agitatoren viel Unheil anrichten.

Zwar versuchte die Hakoah den kleineren Vereinen unter die Arme zu greifen, indem sie ihre Spielstätte zur Verfügung stellte, doch war sie durch die Notwendigkeit, den eigenen teuren Profikader zu unterhalten, selbst in Finanznöten und konnte den befreundeten Vereinen nur bedingt finanziell entgegenkommen. So teilten die kleineren jüdischen Vereine das Schicksal vieler nichtkonfessioneller Vereine, für die es immer schwieriger wurde, den eigenen Spielbetrieb aufrechtzuerhalten. Deshalb schlossen sich kleinere jüdische Vereine in der Folge zusammen und versuchten die Kräfte zu bündeln. An den strukturellen Problemen änderte dies allerdings oft nur wenig. So bildeten mit Kadimah, Hechawer, Hazair-Makkabäa und dem Jewish Sporting Club vier jüdische Vereine, die gemeinsam über rund 500 Mitglieder verfügten, eine Interessensgemeinschaft, um eine eigene Sportanlage zu errichten..$^{50}$ Aus dieser

47 Wiener Morgenzeitung (28. 5. 1924) 10.

48 Wiener Morgenzeitung (1.5. 1923) 10.

49 Wiener Morgenzeitung (1.5. 1923) 10.

50 Wiener Morgenzeitung (16. 5. 1924) 10. 
Interessensgemeinschaft wurde schließlich unter dem Namen JSV Makkabi ein fixer Zusammenschluss. Zwar verfügte der neue Verein in diversen Sektionen von Ballsportarten über Leichtathletik bis zum Wintersport über ein breites Angebot. Über eine eigene Sportanlage verfügte er allerdings - entgegen einer Meldung im Sport-Tagblatt - weiterhin nicht. ${ }^{51}$

Die finanziellen Probleme der Mitglieder hinterließen auch Spuren beim Jüdischen Sportverband, der für seine Tätigkeit auf Zuwendungen seiner Mitgliedervereine angewiesen war. Trotzdem versuchte der Dachverband mit Veranstaltungen - wie einem mehrtägigen Jüdischen Turn- und Sportfest Mitte Juni 1922 am Hakoah-Platz - die eigene Reputation zu steigern. ${ }^{52}$ Auch kam es zur Bildung einer eigenen Verbandsfußballauswahl, gebildet aus Spielern der Hakoah, Hasmonea, Hechawer und Kadimah, die am 25. März 1924 ihre Premiere gegen den erstklassigen ASV Hertha vor 1.500 Zuschauern feierte. ${ }^{53}$

Ein Jahr später, am 20. August 1925, traf die Auswahl - nun auch verstärkt mit Spielern der Grazer Hakoah - im Rahmen eines internationalen jüdischen Turn- und Sportfestes auf ein jüdisches Auswahlteam Palästinas. Das Antreten der Gäste hatten die Verantwortlichen von Hakoah Wien bei ihrer PalästinaTournee im Jänner 1925 fixiert. ${ }^{54}$ Auch mit seinem tschechoslowakischen Pendant entwickelte der jüdische Dachverband einen regelmäßigen Spielverkehr. ${ }^{55}$ $\mathrm{Zu}$ diversen Wettbewerben in Prag entsandte der jüdische Dachverband rund 100 österreichische Aktive. ${ }^{56}$ Auch wurde versucht eine eigene Fußballmeisterschaft für jüdische Vereine zu schaffen, was jedoch daran scheiterte, dass die teilnehmenden Vereine aus der Wiener Verbandsmeisterschaft hätten ausscheiden müssen.

In der Folge gestalte sich der ökonomische Existenzkampf sowohl für Verband als auch für die Vereine immer schwieriger und viele Vereine mussten ihre Tätigkeit einstellen. Neben der Hakoah gab es 1925 nur mehr zehn jüdische Fußballvereine mit rund 1.800 Aktiven. ${ }^{57}$ Die Vereine, die die finanziellen Herausforderungen meistern konnten, wandten sich noch stärker dem Zionismus $\mathrm{zu}$ und wichtige Zionisten übernahmen Präsidentenämter. So wurde der bekannte Arzt Sanel Beer Präsident beim SK Hasmonea, der aber 1936 in die USA

51 Sport-Tagblatt (2. 9. 1924) 4.

52 Wiener Morgenzeitung (5. 4. 1922) 7.

53 Sport-Tagblatt (26. 3. 1924) 2.

54 Sport-Tagblatt (20.8. 1925) 4.

55 Der Morgen (13. 6. 1927) 13.

56 Sport-Tagblatt (9. 6. 1927) 6.

57 Wiener Morgenzeitung (20.10. 1925) 9. 
emigrierte und in Miami als Direktor und Besitzer eines Sanatoriums lebte. ${ }^{58}$ Das gleiche Amt übernahmen Königstein von der Zionistischen Bezirksgruppe Landstraße bei Makkabi oder Dr. Spitzer beim J.A.C. ${ }^{59}$ Zudem hatte die Einführung des Professionalismus, dem zunächst alle jüdischen Vereine bis auf die Hakoah fern blieben, indirekt negative Folgen. Jüdische Vereine, die eine gewisse Spielstärke gewonnen hatten, verloren nun wichtige Stützen an nichtjüdische Mannschaften, die die Herausforderung Profifußball annehmen wollten. ${ }^{60}$

Ende 1925 wurde Dr. Leo Goldhammer, Mitglied des Direktoriums der Zionistischen Organisation Österreichs, Präsident im Jüdischen Turn- und Sportverband, ein klares Zeichen, dass der Verband neben seinen sportlichen Aktivitäten auch die zionistische Arbeit intensivieren wollte. ${ }^{61}$ Doch auch Goldhammer konnte das Auseinanderdriften der Turn- und Sportvereine im Verband nicht beenden und schließlich lösten sich die Fußballer unter ihrem neuen Verbandspräsidenten Emanuel Fiscus von den Turnern ab. ${ }^{62} 1920$ war Fiscus Funktionär beim jüdischen SK Ahawath Zion und wechselte in der Folge zum SK Hasmonea, wo er als Obmann und Leiter der Fußballsektion tätig war. ${ }^{63}$ Beim neuen Jüdischen Sportverband war Fiscus unter anderem als Verbandskapitän für die Zusammenstellung der jüdischen Fußballauswahl zuständig.

1927 musste der J.A.C. aus Ottakring seinen Betrieb einstellen. Dieser Verein gehörte neben der im gleichen Bezirk befindlichen Bar Kochba und der Favoritner Hagibor zu den wenigen Vereinen, die sich außerhalb der Brigittenau und der Leopoldstadt gegründet hatten. Ein Zusammenschluss des J.A.C. mit der Bar Kochba, um die Kräfte zu bündeln, scheiterte. ${ }^{64}$ Andere Vereine mussten den Betrieb einiger Abteilungen einstellen. So löste Kadimah die Fußballabteilung aus finanziellen Gründen auf. In der Folge trat auch die Gewichtheber-Sektion, die einzige noch bei einem jüdischen Verein bestehende, aus dem Hauptverein aus und gründete unter Präsident Dr. Hirsch den jüdischen Arbeiter-Sportklub Cheruth, der von der sozialistisch-zionistischen Vereinigung Poale Zion gefördert wurde. ${ }^{65}$ Die Aktiven von Cheruth sahen ihre Interes-

58 Hugo Gold, Österreichische Juden in der Welt. In: Hugo Gold (Hg.), Geschichte der Juden in Österreich. Ein Gedenkbuch (Tel Aviv 1971) 159-176, hier 160.

59 Wiener Morgenzeitung (5. 4. 1925) 14.

60 Wiener Morgenzeitung (5. 4. 1925) 14.

61 Wiener Morgenzeitung (3.1. 1926) 13.

62 Wiener Morgenzeitung (13. 10. 1926) 8.

63 Wiener Morgenzeitung (18. 6. 1920) 6 sowie (16.12. 1927) 12.

64 Die Neue Welt (21. 10. 1927) 9.

65 Die Neue Welt (15.6. 1928) 10. 
sen vom jüdischen Sportverband nicht angemessen vertreten und schlossen sich der Arbeitersportbewegung an. ${ }^{66}$

Die politischen Grabenkämpfe innerhalb der jüdischen Sportbewegung wurden auch bei der ersten Makkabiade in Tel Aviv im März und April 1932 sichtbar. Diese nach dem Vorbild der Olympischen Spiele organisierten internationalen Sportfestspiele für jüdische Sportler und Sportlerinnen wurden von den jüdischen Arbeitersportvereinen Hapoel boykottiert. Deren Fernbleiben schwächte den Stellenwert der Spiele ungemein. ${ }^{67}$ Trotzdem traten 390 SportlerInnen aus 17 Nationen in Tel Aviv an und Österreich stellte dank der Erfolge der Hakoah-Schwimmerinnen und des Boxers Gustav Laub ${ }^{68}$ hinter Polen die zweitbeste Equipe. ${ }^{69}$ Trotz der massiven politischen Umwälzungen in Deutschland traten bei der Zweiten Makkabiade 1935 in Tel Aviv 1.359 Sportler aus 28 Delegationen, darunter 100 Starter aus Österreich ${ }^{70}$, allesamt von der Wiener Hakoah, an. Die österreichische Delegation konnte sich die Nationenwertung sichern. Parallel dazu wurden bis 1938 zweimal Makkabi-Winterspiele 1933 in Zakopane und 1936 in Banská Bystrica - veranstaltet. Wie schon bei der Premiere trat auch in Banská Bystrica eine eigene österreichische Eishockeyauswahl an. Weil es keinen jüdischen Eishockeyverein gab, wurde sie aus jüdischen Spielern des WAC und der Firmenmannschaft SC Bernhard Altmann gebildet. $^{71}$

Während sich die Hakoah also auch auf internationaler Ebene als das österreichische Aushängeschild des jüdischen Sports präsentierte, kämpften die anderen jüdischen Vereine um ihr wirtschaftliches Überleben. Mitte der 1920erJahre machte der SK Hasmonea eine schwere Vereinskrise durch, als die Zahl der Mitglieder auf 42 Personen sank. ${ }^{72}$ Der Verein drohte aus finanziellen Gründen $\mathrm{zu}$ verschwinden, doch im Gegensatz $\mathrm{zu}$ vielen anderen Vereinen gelang eine Konsolidierung. Ende 1928 war die Zahl der jüdischen Amateurfußballvereine auf vier Klubs gesunken ${ }^{73}$, und als im Juli 1929 der Sportclub Menorah mit dem nichtkonfessionellen Sportclub Fair fusionierte, waren mit Hasmonea,

66 Der jüdische Arbeiter (24. 2. 1933) 6.

67 Haim Kaufmann, Die Makkabi-Spiele. In: Dachs (Hg.), Jüdischer Almanach, 132-141, hier 137.

681930 hatte der Erste Wiener Jüdische Turnverein Makkabi IX eine eigene Boxsektion gegründet, die in der Folge einige österreichische Amateurboxmeister stellte, siehe dazu Die Neue Welt (13. 3. 1931) 11.

69 Kaufmann, Makkabi, 136.

70 Die Stimme (2. 4. 1935) 5.

71 Die Stimme (21. 1. 1936) 6.

72 Die Neue Welt (6. 7. 1928) 7.

73 Die Neue Welt (28.12. 1928) 10. 
Kadimah, die wieder eine Fußballabteilung gegründet hatten, und dem Fusionsklub Hagibor-Makkabi nur mehr drei jüdische Amateurfußballteams vorhanden. ${ }^{74}$ Auch bei den jüdischen Turnvereinen gab es immer wieder ökonomisch bedingte Veränderungen bzw. Zusammenschlüsse. Der Jüdische Turnverein Makkabi XIX ging im Ersten Wiener Jüdischen Turnverein Makkabi IX auf. ${ }^{75}$ Von den diversen kleineren jüdischen Turnvereinen in Wien blieben nur Makkabi II, IX, X sowie XIV übrig. ${ }^{76}$

Noch dramatischer gestaltete sich die Situation außerhalb Wiens: Bis 1930 hatten sich Hakoah Eisenstadt, Hakoah Krems sowie ihr kurzlebiges Leobener Pendant und die Linzer Makkabi aufgelöst. ${ }^{77}$ Abgesehen von kleineren jüdischen Turnvereinen im niederösterreichischen Bereich bestanden nur mehr die Grazer sowie die Innsbrucker Hakoah und der Erste Badener Jüdische Sportclub Unitas. Anfang der 1920er-Jahre hatte sich der Verein mit einer Fußballund Geselligkeitsabteilung unter Obmann Wilhelm Mandl und Präsident Dr. Samuel Spitzer in Baden konstituiert. ${ }^{78}$ Am Beginn der 1930er-Jahre waren österreichweit nur mehr 15 Turn- und Sportvereine übrig. Trotz vieler Versuche war es dem jüdischen Sportverband nicht gelungen, seinen Mitgliedern bei den größten Problemen, den zu geringen finanziellen Einnahmen sowie den fehlenden Spielstätten, nachhaltig unter die Arme zu greifen. ${ }^{79}$

\section{Konsolidierung}

Anfang der 1930er-Jahre gab es nur mehr wenige Vereine, die es schafften, den schwierigen ökonomischen Rahmenbedingungen zu trotzen. Aus der existenzbedrohenden Vereinskrise Mitte der 1920er-Jahre ging der SK Hasmonea gestärkt hervor und zum zehnjährigen Vereinsjubiläum gelang der Fußballabteilung unter der sportlichen Leitung von Alois Grünwald der Aufstieg in die oberste Amateurspielklasse. ${ }^{80}$ Im Zuge einer Zusammenführung zwischen Zweiter Profiliga und höchster Amateurklasse rückte der SK Hasmonea mit

74 Die Neue Welt (19. 7. 1929) 10.

75 Die Stimme (26. 1. 1928) 14.

76 Zum Jüdischen Turnverein Makkabi XV siehe Michael Kofler, Judith Pühringer, Georg Tras$k a$ (Hg.), Das Dreieck meiner Kindheit. Eine jüdische Vorstadtgemeinde in Wien (Wien 2008). 77 Die Neue Welt (1. 8. 1930) 10.

78 Thomas E. Schärf, Jüdisches Leben in Baden. Von den Anfängen bis zur Gegenwart (Wien 2005) 157.

79 Die Neue Welt (6. 3. 1931) 8 und 10.

80 Die Neue Welt (18. 7. 1930) 11. 
zwei weiteren Vereinen für die Spielzeit 1931/32 in die zweithöchste Spielklasse auf. So ließ sich nach der Hakoah mit dem SK Hasmonea ein weiterer jüdischer Klub auf das Risikounternehmen Profifußball ein. Ein wichtiger Beweggrund der zweitklassigen Profivereine, der Aufnahme der neuen Vereine zuzustimmen, war die Tatsache, dass die Aufsteiger über eine entsprechende Anhängerbasis verfügten, die bei Auswärtsspielen Einnahmen in Aussicht stellten. ${ }^{81}$ Aber schon in seiner ersten Profisaison landete der SK Hasmonea abgeschlagen am Tabellenende und musste absteigen. Das finanzielle Wagnis des Profifußballs hatte Spuren hinterlassen und so schloss man sich im Februar 1933 mit dem JSV Makkabi zum Jüdischen Sportverein (JSV) Hasmonea-Makkabi zusammen. ${ }^{82}$ Der neugeschaffene Verein war der letzte noch bestehende jüdische Verein neben der Hakoah, der auch weitere Sektionen wie für Schach, Tennis und Tischtennis unterhielt. ${ }^{83}$

Neben den Fusionen und Betriebseinstellungen gründete sich mit dem Jüdischen Sport- und Schützenverein Menorah - ab Juni 1932 Jüdischer Sportund Schützenverein Haganah - eine neue Organisation, die im 9. Wiener Gemeindebezirk eine Schießstätte betrieb. Seine Mitglieder stammten vorwiegend aus dem studentischen Umfeld. ${ }^{84}$ Der Verein beantragte eine eigene Vereinsuniform mit Vereinsabzeichen ${ }^{85}$ und seine Mitglieder traten selbstbewusst in der Öffentlichkeit auf. So suchten sie nationalsozialistische Veranstaltungen in Wien zu stören. Am 1. Februar 1933 versammelten sich 200 Aktivisten des Vereins im Vereinslokal in der Oberen Donaustraße 89 und störten bei der Salztorbrücke einen nationalsozialistischen Fackelzug, um so Präsenz in der Öffentlichkeit $\mathrm{zu}$ zeigen. ${ }^{86}$

Die Zerschlagung der Republik durch die Regierung Engelbert Dollfuß 1934 hatte, wie für alle gesellschaftspolitischen Bereiche, auch große Konsequenzen für den österreichischen Sportbetrieb. Nach dem bewaffneten Sieg über die Sozialdemokratie in den Februarkämpfen 1934 wurden mit der Sozialdemokratie auch alle Verbände und Vereine der Arbeitersportbewegung verboten. ${ }^{87}$ Auf der anderen Seite des politischen Spektrums wurde nach dem Scheitern des nationalsozialistischen Juli-Putsches im Juli 1934 der Deutsche Turnerbund verboten. Alle verbliebenen legalen Sportverbände und Vereine mussten sich

81 Sport-Tagblatt (21. 7. 1931) 3.

82 Sport-Tagblatt (22. 2. 1933) 3.

83 Die Stimme (7. 4. 1933) 15.

84 Die Neue Welt (27. 3. 1931) 10.

85 ÖStA/AdR, BKA, BP Dion, Jüdischer Sport- und Schützenverein Haganah, XV 999.

86 ÖStA/AdR, BKA, BP Dion, Jüdischer Sport- und Schützenverein Haganah, XV 999.

87 Bunzl, Hoppauf, 23. 
in der Österreichischen Turn- und Sportfront, einer Einheitssportorganisation, zusammenschließen, deren Organisation vielfach in den Händen von Funktionären der christlichsozialen Turnerschaft lag. ${ }^{88}$ Für die überwiegende Mehrheit der jüdischen Vereine, die dem zionistisch-nationalen Lager angehörten, also in Opposition zu linken Vereinen und Organisationen standen, änderte sich nicht viel. Unter der Devise „der Feind meines Feindes ist mein Freund“ schlossen jüdische Organisationen mit dem Austrofaschismus ein labiles Zweckbündnis. Doch im Tagesgeschäft der Vereine hatte dies keine großen Auswirkungen. Antisemitische Angriffe auf jüdische Sportler und Sportlerinnen wurden durch den Systemwechsel nicht unterbunden, ja nahmen in der immer gespannteren politischen Situation noch weiter zu.

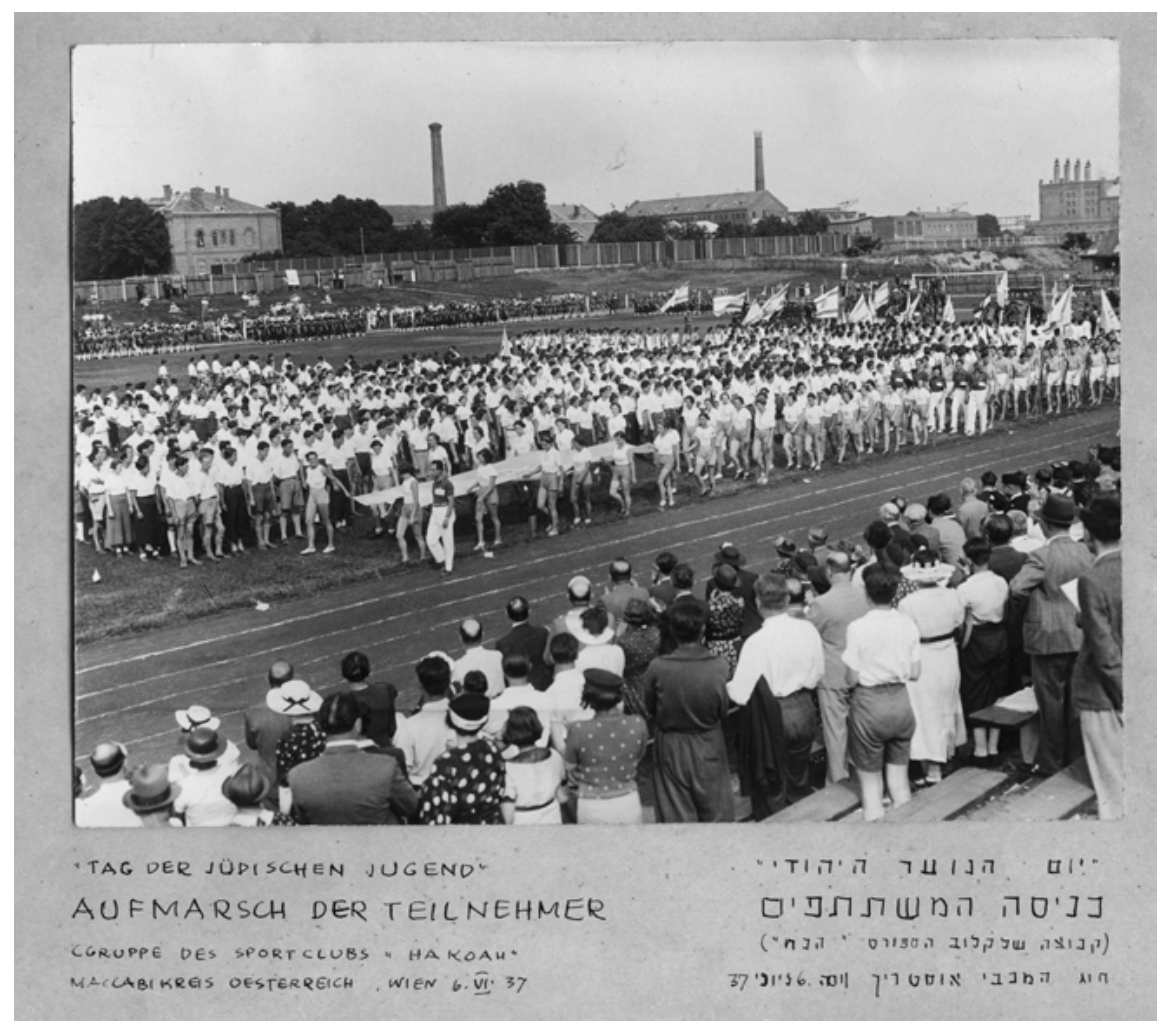

Abb. 6: Tag der jüdischen Jugend, 6. Juni 1937. Parade der TeilnehmerInnen auf dem Hakoah-Sportplatz im Prater (Pierre Gildesgame Maccabi Sports Museum).

88 Bunzl, Hoppauf, 23. 
Risse bekam das Zweckbündnis mit den Olympischen Spielen in Berlin, als der österreichische Makkabikreis den SportlerInnen seiner Mitgliedervereine verbot, an den Spielen im nationalsozialistischen Deutschland teilzunehmen. ${ }^{89}$ Das Antreten jüdischer Vereine aus Österreich in Deutschland war damit aber nicht per se ausgeschlossen. So reisten die Hockey- und Handballabteilung der Wiener Hakoah sowie die Fußballer des JSV Hasmonea-Makkabi Anfang August 1937 auf Einladung des deutschen Makkabikreises zu einer Spielreise nach Deutschland. Trotz der sich weiter verschärfenden Repressalien des nationalsozialistischen Regimes nach Beendigung der Olympischen Spiele 1936 in Berlin konnte der jüdische Sportdachverband Begegnungen in der deutschen Hauptstadt sowie in Hamburg, Leipzig und Frankfurt organisieren. Dies waren die letzten Begegnungen der jüdischen Vereine Deutschlands mit auswärtigen Gegnern.

So erlebten 5.000 Zuschauer auf der Sportanlage im Berliner Grunewald den ersten Auftritt der Wiener gegen ein jüdisches Auswahlteam sowie die entsprechenden Abteilungen der Berliner Bar Kochba. ${ }^{90}$ In Hamburg wurden Begegnungen gegen den dortigen SK Blau-Weiß absolviert ${ }^{91}$, in Leipzig spielten die Wiener gegen eine mitteldeutsche jüdische Auswahl ${ }^{92}$ und in Frankfurt traf man auf die dortige Bar Kochba. ${ }^{93}$

Anfang Jänner 1938 feierte der JSV Hasmonea-Makkabi ein großes Jubiläumsfest im Hotel Metropol am Morzinplatz, ${ }^{94}$ dort, wo sich nach der nationalsozialistischen Machtergreifung die Wiener Gestapo Zentrale befinden sollte. Anfang März 1938 bestanden mit dem Jüdischen Turn- und Sportverband des Kreises Österreich im Makkabi-Weltverband noch ein jüdischer Fachverband sowie 19 Vereine. Verbandsmitglieder waren in Wien der Sportclub Hakoah mit seinen eigenrechtlichen Subvereinen - Fußballklub Hakoah, Schwimmklub Hakoah und Touristik \& Skiklub Hakoah -, der Jüdische Sportverein Hasmonea-Makkabi sowie der Jüdische Sport- und Schützenverein Haganah, der Makkabi Hazair sowie die Jüdischen Turnvereine Makkabi II, IX, X, XV. Außerhalb der Bundeshauptstadt bestanden die Verbandsmitglieder Sportklub Hakoah Graz sowie sein Innsbrucker Pendant, die Makkabi-Turnvereine Groß-Enzersdorf, Mödling, St. Pölten und Wiener Neustadt sowie die beiden Vereine Erster Badener Jüdischer Sportclub „Unitas“ sowie Hapoel Hechadasch. Die beiden

89 Bunzl, Hoppauf, 116.

90 Sport-Tagblatt (2. 8. 1937) 1.

91 Sport-Tagblatt (6. 8. 1937) 1.

92 Sport-Tagblatt (9. 8. 1937) 4.

93 Sport-Tagblatt (11. 8. 1937) 1.

94 Die Stimme (5. 1. 1938) 6. 
Letztgenannten waren die einzigen jüdischen Vereine, die nicht Mitglied des jüdischen Dachverbandes waren. Laut letzter Mitgliederstatistik aus dem Jahre 1936 waren 2.927 Sportler - 1.907 Männer sowie 1.020 Frauen - im jüdischen Dachverband organisiert; die mitgliederstärksten Vereine waren der SC Hakoah mit 780 Mitgliedern sowie der Schwimmklub Hakoah mit 605 Mitgliedern. ${ }^{95}$ Beim Schwimmklub Hakoah war auch das Verhältnis zwischen Männern und Frauen nahezu ausgeglichen. Ein ähnlich ausgeglichenes Verhältnis hatten nur noch der Erste Wiener Jüdische Turnverein Makkabi IX und der Jüdische Turnverein Makkabi XV aufzuweisen. ${ }^{96}$ Für den gesamten Zeitraum von 1899 bis 1938 sind in Wien insgesamt 91 jüdische Sportvereine unterschiedlichster Art nachweisbar.

95 ÖSTA, AdR, ZNsZ Stiko Wien, Mappe 31-N 14, Mitgliederstatistik des Jüdischen Turn- und Sportverbandes Österreichs im Makkabi-Weltverband vom 30. Juni 1936.

96 ÖSTA, Adr, ZNsZ Stiko Wien, Mappe 31-N 14, Mitgliederstatistik des Jüdischen Turn- und Sportverbandes Österreichs im Makkabi-Weltverband vom 30. Juni 1936. 